\title{
Cerebral Ageing
}

\section{ROSSOR}

London

Ageing, the decline in function of the organism from maturation to senescence is a universal feature, and perhaps most poignantly seen in the human central nervous system. The inexorable loss of cells is an integral part of the ageing process, but because neurons cannot be replaced the cerebrum is particularly sensitive to the degenerative effects of ageing. However, within this overall pattern of neuronal disintegration there are examples of selectivity both in the normal ageing process and in the age-related degenerative diseases such as Alzheimer's disease and Parkinson's disease. Evidence of selectivity can be found throughout the central nervous system, and the eye as an embryological evagination from the diencephalon might be expected to share some of these features. A search for such similarities may provide useful clues both to the understanding of the ageing eye and the underlying principles of selective vulnerability within the nervous system.

\section{Patterns of Normal Cerebral Ageing}

Brain weight and volume decline with age. There have been methodological problems in defining this change since the erstwhile maximum brain weight in a given individual is unknown, and social factors may have influenced brain weight in those patients born at the turn of the century compared with those of today. One approach is to measure skull volume which is assumed to remain stable, and to express brain volume as a percentage of the skull volume ${ }^{1}$. Using this technique there is apparent decline in brain volume and brain weight from the age of 60 years onwards ${ }^{1}$. This reduction in brain weight may reflect cell loss or change within the extracellular compartment, but most attention has been directed to changes in the neuronal population. Loss of neurons undoubtedly does occur, and has been consistently shown within the cerebral cortex, and certain subcortical nuclei, using both manual and computerised techniques. ${ }^{2}$ However, the neuronal loss is not a general phenomenon, and certain subcortical nuclei appear to be remarkably stable, with neuron counts in facial nerve nucleus, trochlear nucleus, and the abducens nucleus remaining stable throughout life. Retinal ganglion cells also reduce in number with age ${ }^{3}$ but the nature of this selective vulnerability for the ageing process and the features which might be shared between retinal ganglion cells and cortical neurons, all of which are lost with age, has yet to be determined.

In addition to the demonstration of overall neuronal loss a number of specific characteristics of degenerating neurons have been demonstrated. Lipofuscin accumulates in neurons with age, although certain cells in the brainstem such as pontine nuclei and locus coeruleus are particularly susceptible. Neurofibrillary tangles may also be seen in the hippocampus without clinical features of Alzheimer's disease. A further feature of degenerating neurons is attrition of the dendritic tree. This has been elegantly demonstrated using the Golgi silver technique which stains the entire cell body and neuronal processes. This technique stains only some neurons in a given section and can thus provide considerable detail of a few individual cells. Within normal ageing there appears to be a loss of neuronal processes in many neurons $^{4}$ (Figs. 1 and 2 ) but surviving neurons do demonstrate some plasticity with increased growth of the dendritic tree. ${ }^{5}$ The molecular basis of the selectivity of Golgi staining is not

From National Hospital for Nervous Diseases, Queen Square, London WC1 and Department of Neurology, St Mary's Hospital, Praed Street, London W2.

Correspondence to: Dr M. Rossor, Consultant Neurologist, St Mary's Hospital, Praed Street, London W2. 

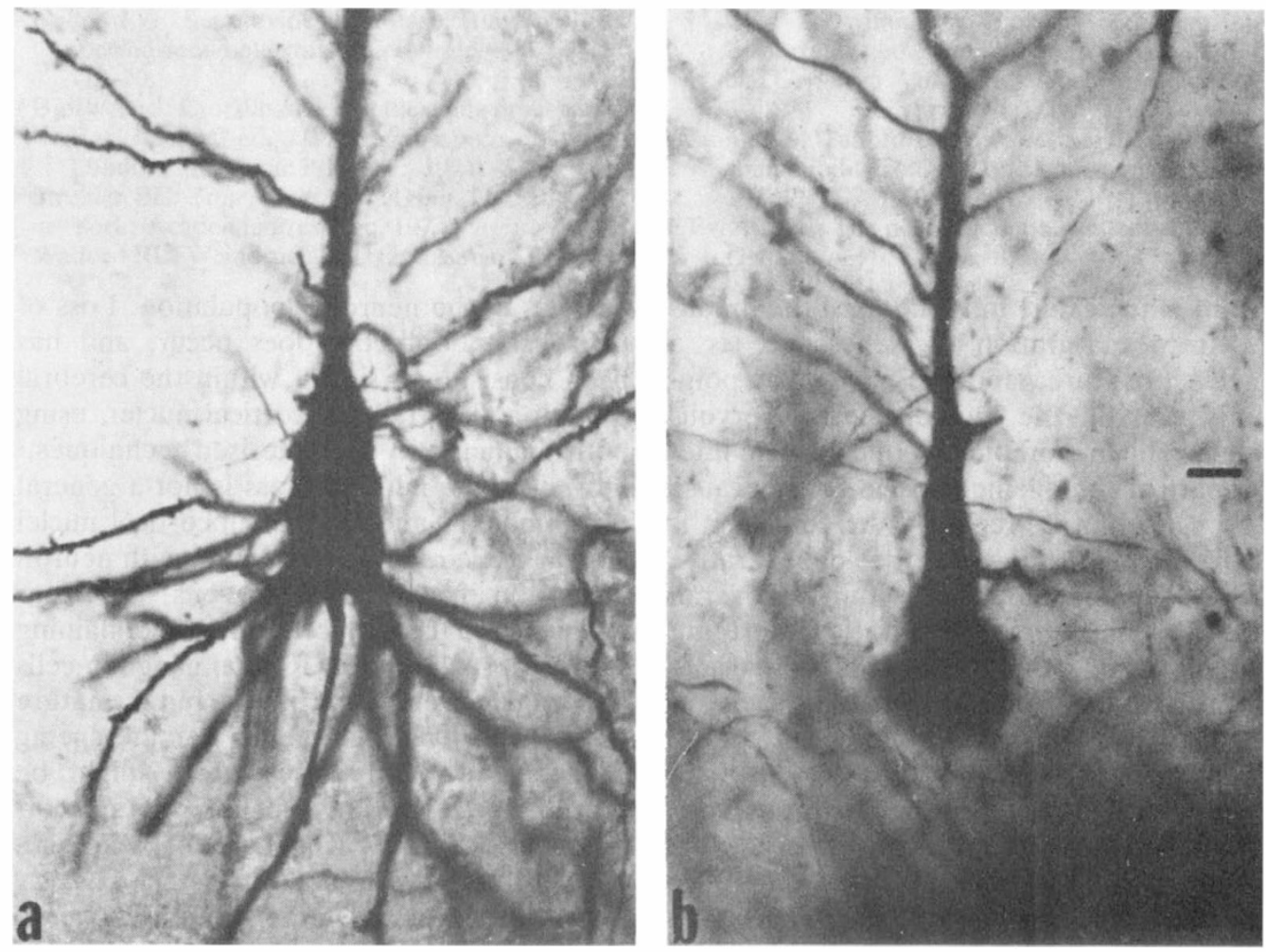

Fig. 1. Golgi preparation of giant motor pyramidal cell of Betz (a) adult mature cell (b) degenerative change showing loss of basilar dendrites Calibration bar $10 \mathrm{~m} / \mu$. Reproduced with permission from Scheibel Ref (4).

known and thus whether the observed changes in dendrites affect all neurons is also unclear. The extent to which dendritic processes may alter and the presence of plasticity with age in retinal ganglion cells and its implications for function has yet to be determined.

The basis of the selectivity of neuronal loss and degeneration with normal ageing is unknown. Considerable attention, however, has been directed towards the neurotransmitters that these cells may utilise. There is now good evidence for the selective involvement of defined neurotransmitter systems in certain CNS degenerative diseases, for example Parkinson's disease, but data on normal ageing is less secure. The concentrations of some neurotransmitters such as GABA show clear reductions with age, others, however, show greater stability. For example, choline acetyltransferase, the cholinergic marker enzyme, declines in frontal cortex but shows remarkable stability in other areas of the cerebral cortex. Concentrations of somatostatin, one of the intrinsic cortical peptides, also appear stable throughout life. ${ }^{6}$ The delineation of the neurotransmitter circuity of the retina and the demonstration of a number of peptides both within the retina and within the innervation of the uveal tract presents the opportunity of assessing whether similar selectivity occurs within the eye.

Parkinson's Disease and Alzheimer's Disease Parkinson's disease and Alzheimer's disease relate closely to the question of cerebral ageing. Both are diseases of senescence, and the neurofibrillary tangles of Alzheimer's disease can be found in the hippocampus of normal old age. The characteristic biochemical feature of Parkinson's disease is a dopamine loss consequent upon nigrostriatal damage. A characteristic although not exclusive, feature of Alzheimer's disease is a cholinergic deficit in the cerebral projection system. In addition 

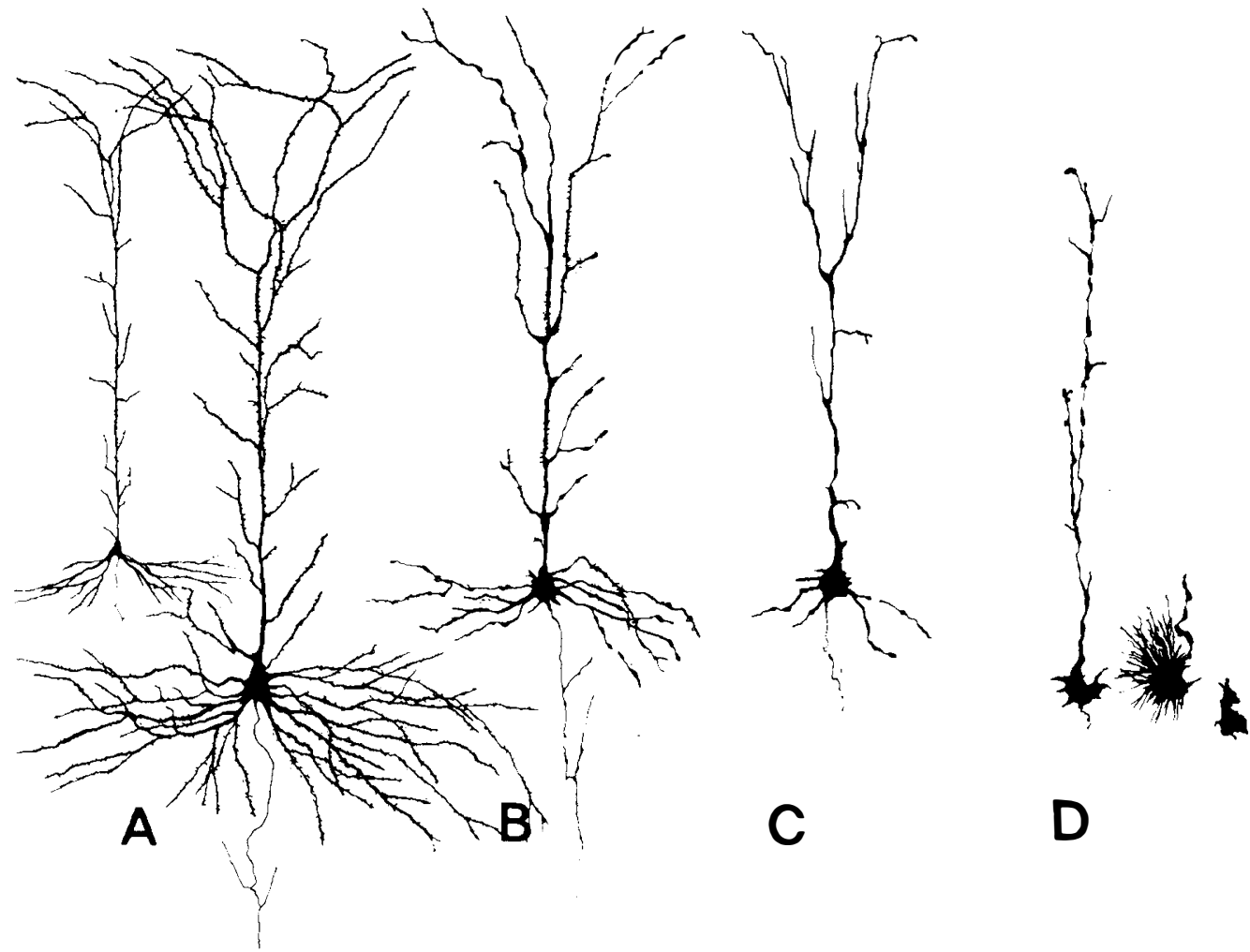

D

Fig. 2. Drawing of neocortical pyramidal cells showing sequence of age-related changes starting with mature, fully developed pyramids in $(A)$ and progressing through alterations characteristic of the senium $(B$ and $C)$ to final stages of cell dissolution (D). Based on Golgi stained material and drawn at $\times 200$. Reproduced with permission from Scheibel Ref (4).

to the possible involvement of the cortical visual system in these diseases the presence of both dopaminergic and cholinergic amacrine cells in the retina prompts the search for abnormalities in the visual system associated with these disorders.

In addition to the nigrostriatal system there is also a dopamine projection to the cortex and limbic structures, and dopamine within the hypothalamus; abnormalities in these areas have been noted in Parkinson's disease. Abnormalities in visual evoked responses, most commonly interocular asymmetries which resolve following levadopa therapy have been reported but there are conflicting studies. ${ }^{7}$ The author is unaware of published data on retinal dopamine in Parkinson's disease and immunohistochemical studies would be of considerable interest. The reduction in choline acetyltransferase activity in Alzheimer's disease is found throughout the cerebral cortex, and includes the visual area. It is not, however, a universal feature of cholinergic cells, since there is definite sparing of, for example, the putamen and red nucleus, and it is now seen as part of a wider spectrum of abnormalities. ${ }^{8}$ The abnormality in the cortical cholinergic system relates to damage to a diffuse ascending projection from the basal forebrain, which may have a tonic influence on cerebral function, rather than damage to intrinsic cholinergic "hard wiring". Cholinergic amacrine cells are found in the retina, but there are no available published data on cholinergic function in the retina in Alzheimer's disease. Patients may develop problems of visual agnosia which can be difficult to dissect out from the general dementing illness, and primary visual disturbance has not been a prominent feature of the disease. Abnormalities in contrast sensitivity function would suggest that early processing is normal, 
but later processing within the cortex is abnormal. ${ }^{9}$ However, testing of demented patients is very difficult and results need to be interpreted with caution. A recent histological study demonstrated a loss of fibres within the optic nerve $;{ }^{10}$ this is an intriguing observation that remains to be confirmed, but prompts immediate questions of shared vulnerability with cerebral cortex.

In summary, the retina as specialised neural tissue derived embryonically from the diencephalon presents a valuable model for analysing the effects of neuronal disintegration both the normal ageing and in age-related degenerative disease. The study of this model may assist in the understanding of the decline in visual function with age and with determining the molecular basis of selective vulnerability.

\section{References}

${ }^{1}$ Davis PJM and Wright EA: A new method for measuring cranial cavity volume and its application to the assessment of cerebral atrophy at autopsy. Neuropath. Appl. Neurobiol. 1977, 3: 341-58.

2 Tomlinson BE: The Structural and Quantitative
Aspects of the Dementias. In Roberts PJ ed Biochemistry of Dementia. John Wiley and Sons, 1980, pp 15-52.

${ }^{3}$ Weale RA: A Biography of the Eye. H K Lewis, London. 1982.

${ }^{4}$ Scheibel AB: Some neural substrates of ageing and senescence in the central nervous system. In Issaacs B ed Recent Advances in Geriatric Medicine, Vol 2. Churchill Livingstone 1982, pp 147-57.

${ }^{5}$ Buell SJ and Coleman PD: Dendritic growth in the aged human brain and failure of growth in senile dementia. Science 1979, 206: 854-6.

${ }^{6}$ Rossor MN, Iversen LL, Reynolds GP, Mountjoy $\mathrm{CQ}$ and Roth M: Neurochemical characteristics of early and late onset types of Alzheimer's disease. Br. Med. J. 1984, 288: 961-4.

7 Bodis-Wollner I, Yahr MD and Mylin LH: Nonmotor functions of the basal ganglia. In Hassler RG and Christ JF eds Advances in Neurology Vol 40, Raven Press, New York, 1984, pp 28998.

${ }^{8}$ Rossor $M$ and Iversen LL: Non-cholinergic neurotransmitter abnormalities in Alzheimer's disease. Br. Med. Bull. 1986, 42: (1)70-4.

${ }^{9}$ Schlotterer G, Moscovitch $\mathbf{M}$ and CrapperMcLachlan D: Visual processing deficits as assessed by spatial frequency contrast sensitivity and backward masking in normal ageing and Alzheimer's disease. Brain 1983, 107: 309-25.

${ }^{10}$ Hinton DR, Sadun AA, Blanks JC and Miller CA: Optic-nerve degeneration in Alzheimer's disease. New Eng. J. Med. 1986, 315: 485-7. 